\begin{tabular}{|c|c|c|}
\hline 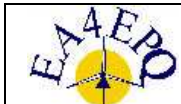 & $\begin{array}{l}\text { European Association for the } \\
\text { Development of Renewable Energies, Environment } \\
\text { and Power Quality (EA4EPQ) }\end{array}$ & $\begin{array}{l}\text { International Conference on Renewable Energies and Power Quality } \\
\text { (ICREPQ'12) } \\
\text { Santiago de Compostela (Spain), 28th to 30th March, } 2012\end{array}$ \\
\hline
\end{tabular}

\title{
Use of the Power Line Communication System (PLC) at Low Voltage (0.4 kV) Noisy Electrical Networks - Introducing a New Concept at Power Quality
}

\author{
István SZÉN ${ }^{1}$, Ervin RÁCZ $(\mathrm{PhD})^{1}$ \\ ${ }^{1}$ Institute of Power Engineering \\ Kandó Kálmán Faculty of Electrical Engineering, Óbuda University \\ Bécsi út 94-96A, 1039 Budapest (Hungary) \\ Phone/Fax number:+0036/1-666-5828, \\ e-mail: szen.istvan@kvk.uni-obuda.hu \\ e-mail: racz.ervin@kvk.uni-obuda.hu
}

\begin{abstract}
Micro grids, smart grids, small power plants in range of household size stay in the lime-light. Nowadays, smart metering system stays directly before installation. All of the above mentioned systems require complex and discrete concepts and knowledge. Definitions and use of the right concepts and correct knowledge belong to actual questions of energetics. Small size distributed power plant units, drivers, controllers and switches belong to the power plants connecting to power grids can introduce high-frequency noises. In this work the spectra and the impact of the high-frequency noises have been investigated. After analysing the measured spectra followings can be declared: High-frequency noises can trouble the Power Line Communication (PLC) system at smart metering systems. Noise can destroy propagating parameters of the useful signal affecting the safety of data communication.
\end{abstract}

\section{Key words}

Power Line Communication, S-FSK, OFDM, Direct Line Communication, Smart Metering, Smart Grid, Power Quality, Network Quality.

\section{Introduction}

Electric energy is a product. As an important product electric energy must satisfy well defined quality trends. The quality trends are regulated by laws and rules in the nations around the world. Nowadays, countries improve their electrical systems. One way of improvement is introducing and building smart grids at power systems. Installing smart grids, micro grids and implementing other state of the art power systems into electrical systems definitely improve their quality. It is clear that, we need to count with appearing of renewable energy sources, mini power plants, and household-size power plants next to greater size basic power plants. As an example figure 1. shows the distribution or ratios of every household-size power plants installed at different supplier companies such as ELMÜ-ÉMÁSZ, E-ON, and EDF-DÉMÁSZ in Hungary in year of 2011.

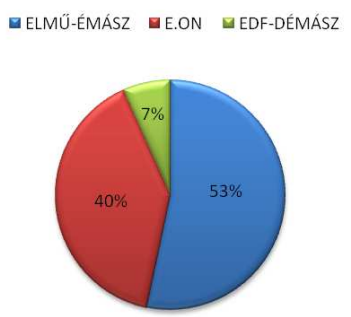

Fig. 1. Distribution of all household-size power plants installed at different supplier companies such as ELMÜ-ÉMÁSZ, E.ON and EDF-DÉMÁSZ in Hungary in year of 2011.

Mentioning an other example figure 2. draws what kind of renewable energy sources were installed into the civil Hungarian power systems by the first half of 2011 .

Installing of these new energy sources new challenges will be outcroped. New challenges must be solved by electrical engineers and other professionals. For example, it is important to know that how these small power plants connect to the central power system. Parallel to the advantages of the small systems they can introduce significant electric noises in the central electric system. Figure 3. shows an example how a mini solar cell power supply system connect to a $0.4 \mathrm{kV}$ main central electric line.

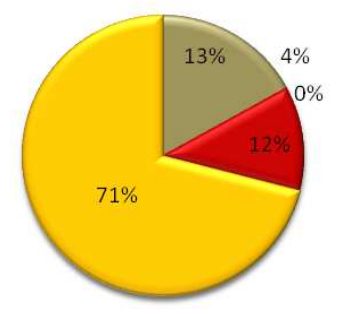

Fig. 2. Ratios of the installed renewable energy sources - solar energy (yellow), wind energy (red) and bio energy (brown) - in the Hungarian civil power system by the first half of 2011.

It clearly can be seen from Figure 3. that the mini solar power supply system connect to the central line through $\mathrm{AC} / \mathrm{DC}$ inverter and a transformer (as main parts). If the mini system sends electric noises into the main power line, in this case the possible source of the noise must be searched. In other words, investigation of the solar cells, $\mathrm{AC} / \mathrm{DC}$ inverters and transformers are necessary. 


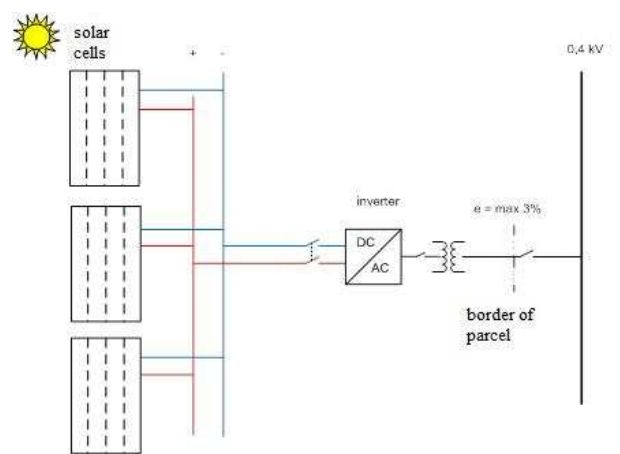

Fig. 3. Connection scheme of an ideal mini solar power supply to a central $0.4 \mathrm{kV}$ power line. $[1,2]$

In the recent years some new energy power supply concepts have been introduced. However, there are open questions around these new ideas. Answering these open questions will be crucial tasks for the near future. One of these results or concepts introduced in the recent years is smart grid. The smart grid integrates communication and power supply networks in one with their advantages and disadvantages together. In other words, the smart grid contains some smart and useful functions from communication and power source networks. These functions can be combined with each other resulting complex picture of the smart grid output.

Not only the suppliers but consumers are also motivated at energy use: customers want to use less energy. Practising economy at energy use is key parameter in our present world. Economy around the world is collapsing or shaking almost everywhere. Using energy smartly is key function of human thoughts. In order to try to save energy a simple user or customer use to purchase economy friendly electric household goods (for example: energy saver light bulbs). Unfortunately, consumers do not think about profession of electrical engineering during their purchase. Buying some cheap or low quality parts may generate more problems. One of the important problems are electric noise. In other words, people may buy very noisy products (noise means electrical noise in this fact) as well. These noisy products can send electrical noise signals with higher frequency to the electrical system causing many problems for the supplier side. Companies as suppliers try to fix these problems.

Nowadays, not only the noisy electrical products but some new energy sources with considerable electric noise production also appear in the low voltage network. For example some renewable energy sources and new generator units may be significant sources of electrical noises. (For example figure 3. draws how a mini solar cell power supply connects to the central electric system.) It is also known that some renewable energy sources pollute low voltage electric grids with harmonics. Noises pollute electrical systems. Communication systems of the smart grid can be shown incorrect operation due to unnecessary electrical noises. It is clear that both communication functions and expansions of smart grids have required new approach in the field of power quality.
In order to monitor, measure and continuously check the smart electrical grids a smart metering system has been introduced. The smart metering system contains smart meters. One of the most important parts in the smart metering system is the power line communication (PLC) subsystem. It means that if the PLC does not work properly, it results incorrect operation at the whole smart metering system.

PLCs are very sensitive for electric noises. Unfortunately, high frequency electric noises can affect their operations. The power line communication works at frequency range of $9-95 \mathrm{kHz}$ (CENELEC A-band). This frequency range is endangered by electric noises. Another important factor is: the PLCs cannot be substituted by other parts. They must be protected!

The aim of the present research work was mapping the noise effect on PLCs. In order to investigate the role of the high frequency noises on PLC operation, some test measurements on PLCs with different inverters have been made. Figure 4. presents the actual experimental setup used for the measurements.

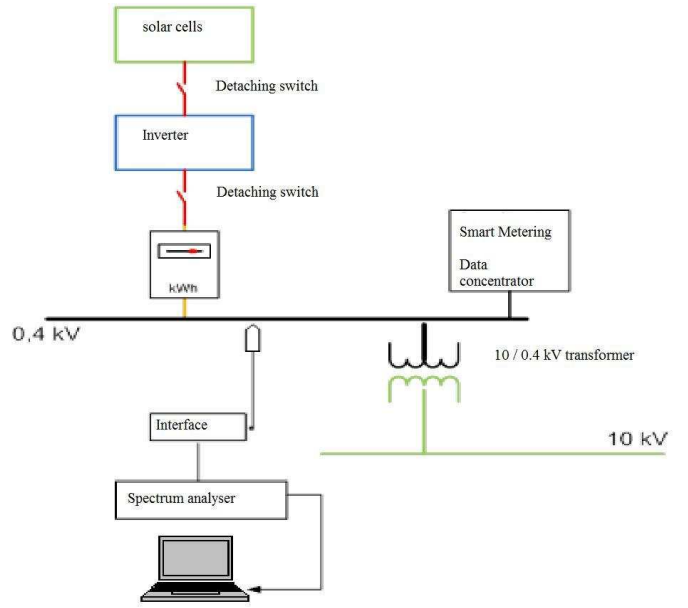

Fig. 4. Experimental setup for tests measurements. Different inverters have been tested. [3]

Basically, PLC investigations can be divided into two parts. In the first case high frequency propagation specifications of standard low voltage wires have been investigated in laboratory. Later, all data have been checked by real field measurements as well. Finally, disturbance keeping and tolerance of PLC have been tested at real circumstances. Using our current results we plan to compare modulations of S-FSK and OFDM in year 2012. This way, evaluation of our results can be done by complex techniques.

\section{Investigation of propagation specifications}

In Europe lots of modulation techniques are being used but only several modulation modes can be used at Smart Metering. Information content of all examined signals showed by amplitude, frequency, phase plots or variation of these parameters. Number of carrier frequencies are also important at reliability of communications. 
Nowadays, in Hungary the Spread-Frequency Shift Keying (S-FSK) is the mostly used unique method. Following trends of manufacturers probably in year 2012 new meters will be introduced based on new modulations. In opinion of our team Orthogonal Frequency-Division Multiplexing modulation technique (OFDM) will be available for us both for real field and laboratory measurements. This modulation technique has two developments: they are PRIME and $3 \mathrm{G}$. It is planned to investigate and compare PRIME and 3G systems in 2012. In same year deep scan of Direct Line Communication (DLC) is also scheduled.

\subsection{Skin - effect}

In case of standard low voltage supply of electric energy $(0.4 \mathrm{kV}, 50 \mathrm{~Hz})$ knowing the resistance (according to Ohm's law) of all wires or cables gives us enough information for our work. Using PLC technique (CENELEC A-band) the standard concept of resistance must be redefined, and new specifications and consequences belong to new functions must be taken into consideration.

It is well known that, $\mathrm{AC}$ resistance of a given conductor is higher than DC resistance of the same matter. Resistance of a given wire and current displacement increase with the working frequency. By increasing the frequency of the electric current, conducting electrons try to wander to the outer shell of the conductor. Electron density will be higher - generating like a skin - close to the outer shell of the matter. This is the well-known skin effect.

Due to PLC technique used cables must be rechecked in 9$95 \mathrm{kHz}$ frequency range. During the checking period an optimal length of cable could be discovered. Using the optimal length of cable all PLC signals can be transmitted keeping their good quality. Theoretical calculations show that, possible optimal lengths of cable may fall between several hundred and thousand meters $(700 \mathrm{~m}-7500 \mathrm{~m})$. However, taking into account additional parameters such as material of the cables, recommendations of the manufacturers of measuring tools, size of zones of energy supplying, etc., these parameters can modify the estimated length of cables.

\subsection{Crosstalk}

In laboratory single-phase flow meters have been tested, but during field measurements three-phase flow meters have been installed particularly. During install of threephase flow meters an interesting effect was detected: the crosstalk.

At the installed smart metering system all flow meters give PLC signal at the phase \#1. (This is the default configuration given by the manufacturers.) During the field measurements we observed that, same data or information signals were detected at cables of phase \#1., phase \#2. and phase \#3 (three-phase system). In other words, strong crosstalk effect was observed between all single-phase cables. The crosstalk effect proved that high frequency signals could cross-induced from a single-phase cable to another phase. Knowing this effect is crucially important at our measurements.

\subsection{Reflection, attenuation of signals}

In the case if the impedance changes during propagation direction of the PLC signal two effects can be appeared: the given signal can be reflected in the cable or any distortions of the signal shape can be detected. Unfortunately, the mentioned distortion and reflection effects can be observed in other cases as well. For example: in case of non-constant cable cross section or diameter both above mentioned effects can be observed. Situation is similar if there are cable connections (or in worst case there are bad quality cable connections) on the way of propagation of the signals. In this case, reflection or signal distortion effects may be strong. Strength of the distortion effects may depend on the quality of the cable connection.

\section{Vulnerability of the PLC signal; disturbance keeping by consumers}

Propagation of the signal used for transmitting information can be influenced by the above mentioned parameters, but role of some other factors also can not be neglected.

At the beginning of this paper, it has been shown that, if we would like to input electrical energy into a public electrical power system one of the most important factors of the electrical power production is inverter. In other words, inverter can not be neglected from any electrical power production systems.

Basically, inverter is a nonlinear element of electrical circuit. Operation of it causes generation of high order harmonics at the circuit system. This effect can decrease efficiency of the signal transmission of a PLC. Some inverter products have been made by manufacturers according to European standards. These products are reliable in European systems and their system noise reduction is an important parameter. However, in case of some other products the system noise reduction is unfortunately neglected technical parameter.

Traditional end-consumer products (for example energy efficient light bulbs, dimmer lamps, microwave ovens, etc...) can drastically influence the PLC signals. During our measurements we were able to prove that electrical products used in personal households are source of noise at the frequency range of PLC operation.

Figure 5. shows a PLC activity during the field measurement of our experimental series. Studying plot of Figure 5. two carrier frequencies can be observed at 79 $\mathrm{kHz}$ and $90 \mathrm{kHz}$. Measurement range was a CENELEC A-band. 


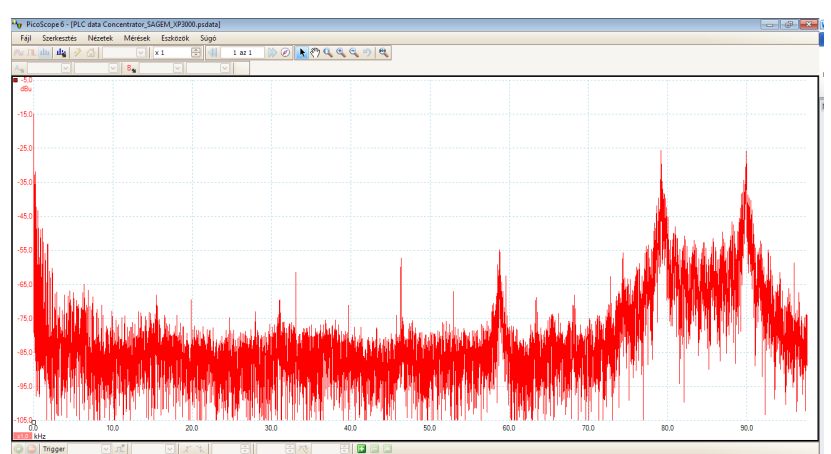

Fig. 5: PLC (S-FSK) activity with two carrier frequencies at $79 \mathrm{kHz}$ and $90 \mathrm{kHz}$ [3].

Figure 6. presents a PLC spectrum signal during an energy efficient fluorescent lamp was operated. The energy efficient fluorescent lamp can be purchased in commercial shops and its price is not too high. Due to its realtively low price it became a popular product in households. Figure 6 . clearly shows that operation of the energy efficient fluorescent lamp can strongly disturb the PLC signal at the monitored frequency range. During operation of the fluorescent lamp connection between data concentrator device and flowmeter was disturbed, and the reliability of the data monitoring was also decreased.

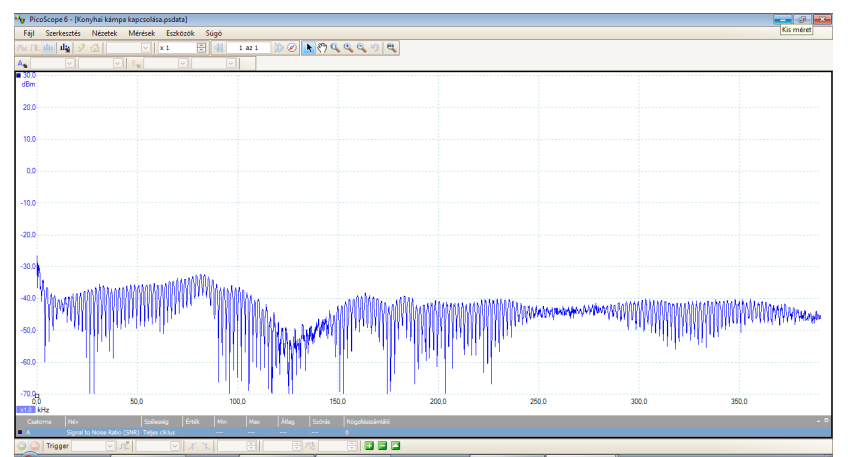

Fig. 6.: PLC spectral feature saved during operation of an energy efficient fluorescent lamp showed in the frequency range of $9-95 \mathrm{kHz}[3]$.

Figure 7. shows a similar measurement. The figure presents a PLC data signal saved during operation of a microwave oven. Figure 8. presents another PLC signal during operation of a dimmer light bulb. In all examined cases decreasing the reliability of the PLC signal was clearly observed. It can be presumed that, at noisy conditions reliability of the PLC signal can be improved by increasing the number of carrier frequencies. In year 2012 it is planned to improve our experiments and setups in order to prove our observations.

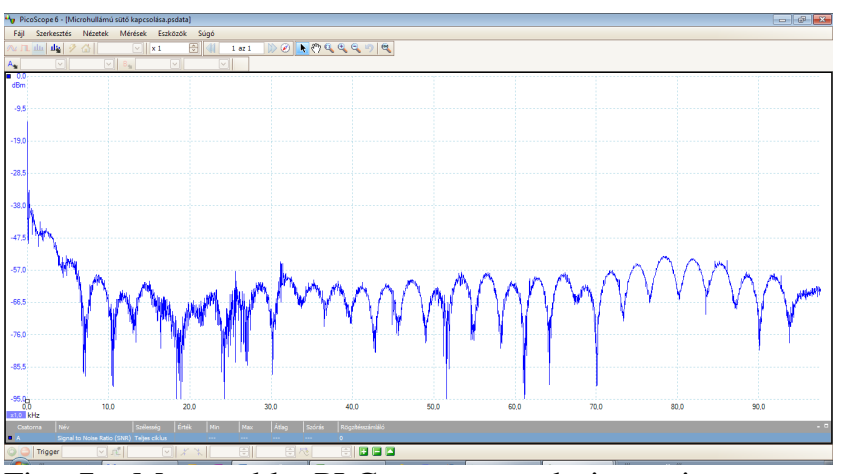

Fig. 7.: Measurable PLC spectrum during microwave oven was operated. [3]

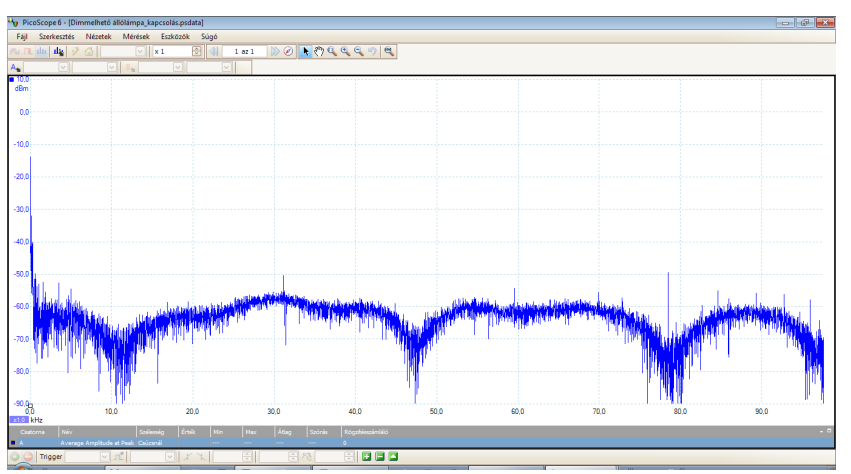

Fig. 8.: Observed PLC spectrum during dimmer light was operated. [3]

\section{Propagation of PLC signal during field measurements}

Experiments and investigations were made at real field measurements sponsored and helped by Budapesti Elektromos Müvek Zrt (Budapest Electric Company, LLC).

Our experiments were made at two type of households. In the first case, relative small size electric grids with many consumers belonged to the household were investigated. This type of household - household \#1 - represents typical Hungarian apartment structure in an apartment house or apartment building or tower house. Second type of households - household \#2 - were family houses with gardens at suburban area. A single household \#2 typically has big size electric grid system with low number of consumers.

\subsection{Propagation of PLC signal in case of Household \#1}

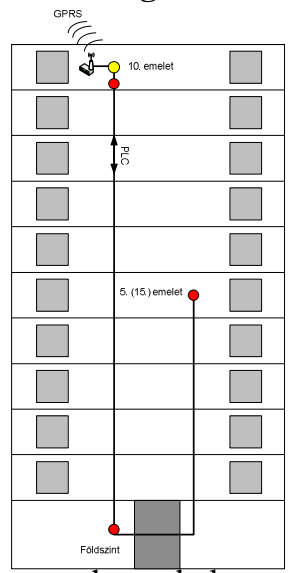

Fig. 9.: Measurement scheme belongs to measurements at household \#1. [4] 
Figure 9. shows that data concentrator device was installed on the top floor of the apartment building (floor 10, see Fig 9). Selected measurement positions are signed by red dots. First measurements were made close to the data concentrator on floor 10 . This measurement played as role of reference measurement. Measurements were continued on the ground floor, and finally experiments were finished on the fifth floor. In the second and the third case distances between the data concentrator and the measurement positions were measured as around 30 and 45 meters, respectively. Main configuration of the measurement system can be studied in figure 9 .

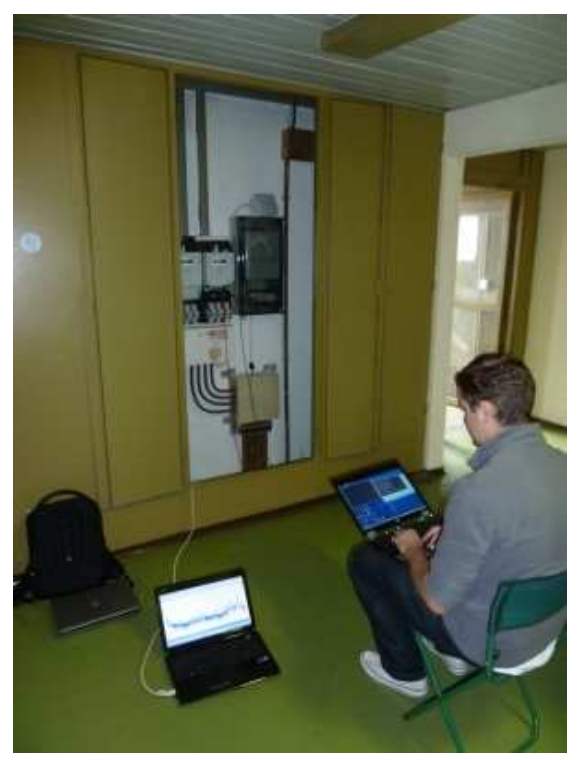

Fig. 10: Photograph showing the PLC measuremet on floor 10 close to the data concentrator at household type \#1. [4]

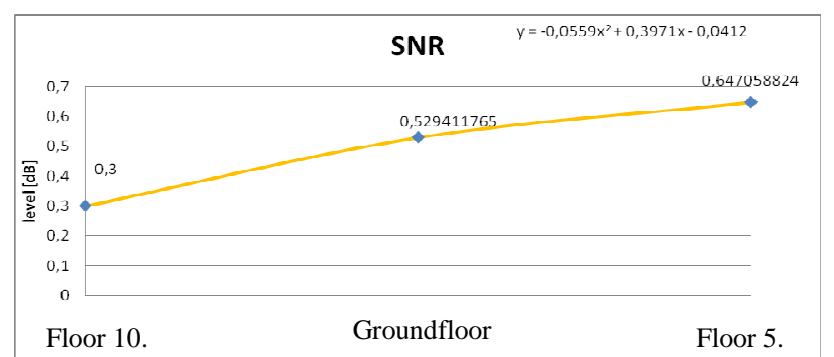

Fig. 11.: Signal - noise ratio (SNR) of the measured data during the PLC measurements at household type \#1. [4]

Figure 11. presents signal - noise ratio (SNR) during PLC measurements at household type \#1. As Figure 11. shows SNR value is 0.3 on floor 10 close to the data concentrator device. Valuse of SNR increases with measured distance between data concentrator device and measurement position. Longer distance generated higher SNR values. For example: if the distance was 45 meters originated from the concentrator, the SNR value was close to 0.7.

\section{Experiences:}

- In case of higher number of consumers significant electric noises were generated. In other words, in case of higher number of consumers the electric noise can not be neglected.
- Appearing of electric noises showed stohastical behaviour with frequently occuring events.

- Cables and wires are parallel to each others on the full length of the resistance range. Due to this topology, crosstalk appears at all frequency phase.

- Slow data connections: $0.58-0.63 \mathrm{kbps}$

- Read out of the load curve was too slow. It was taken for long time.

Recommendation:

- In case of households type \#1 (apartments in apartment houses) use of typical serial communication (RS 422) or Ethernet system can be introduced successfully.

\subsection{Propagation of PLC signal in case of Household $\# 2$}

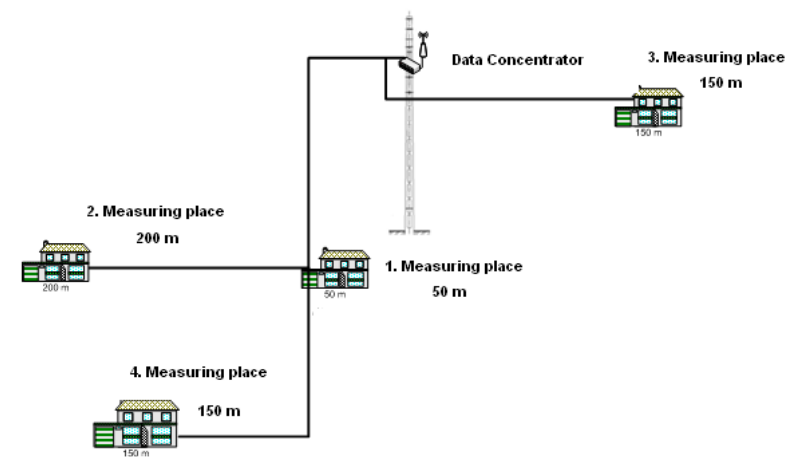

Fig. 11.: Geometry of our measurements were made at suburban area at family houses with gardens. [4]

Measurements were started at flow meter installed closest to the data concentrator. This flow meter was installed 50 meters far from the data concentrator device. Next measurements were made 200 meters, than 150 meters and than another 150 meters far at different direction from the data concentrator, respectively.

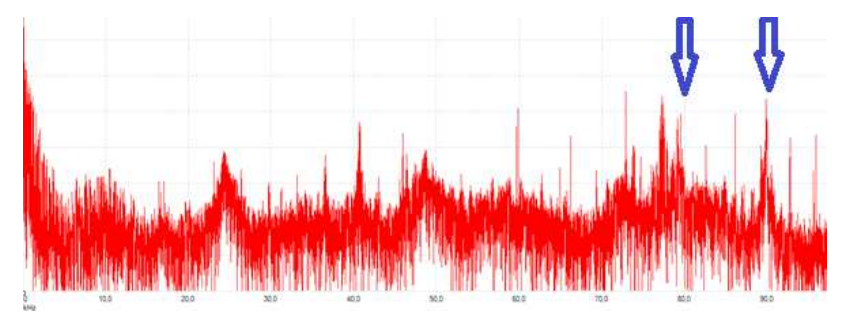

Fig. 12.: Measurement data shows significant noise level at PLC region in case of measurement at household \#2. Carriers can not be observed due to strong noise level. Finally, communication resulted incorrect transmission of information and interrupted connection (measurement position \#4). [4] 


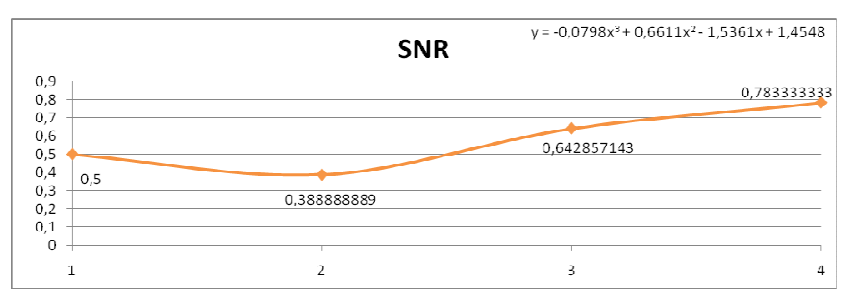

Fig. 13.: Development of signal - noise ratio (SNR) during measurement at household \#2 (family houses at suburban area) [4].

\section{Experiences:}

During our experiments main experiences were the followings: connections and reliability of the flow meters depend on the distance measured between the data collector and the flow meters. Efficiencies of the PLC communications are significantly influenced by the numbers of the household devices used, and by their operations and their timing of operation. SNR values fell between $0.4-0.8$ during the experiments. Another observation was: this type of environment was inhomogenious. Measurements at apartment building showed more homogenious behaviour. Running our experiments at same experimental position signal - noise diagram showed fluctuations causing statistical errors. Signal - noise ratio depends on the daylight time period. For example: afternoons and night times stronger noises were detected than in the morning. Weekends and in the night periods noise signals were significantly stronger influecting read out cycle of flow meters.

Other experiences:

- $\quad$ Relative big geometrical area (field measurement) was investigated (50 - 300 meters).

- Lower number of consumers connected to bigger geometrical area.

- Consumer products are inhomogenious. (Used, old or brand new devices were operated; living time at home building).

- Sometimes - mainly night times and weekends availability of the devices and flow meters were poor.

- Smart meters have puffer capacity, therefore periodically upcoming noise signals do not generate problems.

- Speed of signal propagation were measured between $0.68-0.79 \mathrm{kbps}$.

- Registers read outs were fast, but load curves read out were relatively slow.

\section{Conclusions}

- MSZ EN 50160 standard must be reviewed; opening a new chapter would be necessary, because public electrical energy grid had been extended with new function (communication channel).

- Grid analysis measurement to $50^{\text {th }}$ high harmonics is not enough. It is necessary to investigate the range of $9-95 \mathrm{kHz}$ as well.

- During selection of cables and wires high frequency specifications and features must be taken into account.

- During designing, building or reconstructing the electrical grid systems impedance fitting (relexion, signal amplitude decreasing) must be taken into account.

- At concentrated consumer places (for example apartment buildings, tower buildings or groups of tower buildings) it is recommended to investigate the following: whether do we need PLC communication? (Investigating both technical and economical viewpoints.)

- At concentrated consumer places use of Ethernet or RS-422 connections are recommended.

- After analysing and evaluating our experimental data we can declare the following: S-FSK modulation is outdated. Use of OFDM modulation (more carrier frequencies) is recommended. Using OFDM modulation reliability of communication will be improved.

\section{References}

[1] Dr. Morva György, Szén István (BMF KVK VEI): Connections of small power plants (Research Report)

[2] Dr. Morva György, Szén István, Zakár István (BMF KVK VEI): Distribution grid connections of household size small power plants III. BMF Energetics Conference, 2008., November 25, Budapest

[3] Szén István: Smart Metering - Scientific investigation of the propagating parameters of PLC. (Scientific Report), 2011. July 12.

[4] István Szén: Smart Metering and PLC - (Scientific Report) 2011. December 9.

[5] Ersan Kabalci, Yasin Kabalci, Ibrahim Develi, - Modelling and analysis of a power line communication system with QPSK modem for renewable smart grids - Electrical Power and Energy Systems 18 April 2011 